\title{
Lessons from Rinderpest Eradication with Reference to COVID-19 Pandemic Management
}

\author{
Martyn Jeggo ${ }^{1}$ and Peter Roeder ${ }^{2}$ \\ ${ }^{1}$ GCEID, Deakin University, Geelong, Australia \\ ${ }^{2}$ Taurus Animal Health, Hampshire GU35 8SY, UK
}

\section{RINDERPEST-THE DISEASE}

Rinderpest (otherwise known as cattle plague) was caused by a morbillivirus of the family Paramyxoviridae. Viruses belonging to this family cause disease in many different species, including measles in man. Rinderpest is marked by fever with ocular and nasal discharges and can cause high morbidity and mortality rates from oral and gastrointestinal tract ulceration, diarrhoea, dysentery, dehydration, protein loss, and immunosuppression resulting from lymphocyte depletion.

For millennia, rinderpest was the scourge of livestock farmers spreading repeatedly out from its central Asian origin into east and south-east Asia and westwards into Europe, its impact changing the course of human history. From the late 19th century, when it was introduced to Africa, it devastated both domesticated livestock and wildlife whilst continuing to generate devastating pandemics in Asia. Not surprisingly, its control dominated the activities of veterinary services from their foundation in the 18th century into the late 20th century (Roeder et al 2013).

Martyn Jeggo: Former member of GREP, managing the rinderpest laboratory network, Peter Roeder: Former Secretary of GREP.

Published online: September 15, 2021

Correspondence to: Martyn Jeggo, e-mail: jeggo.martyn@gmail.com

\section{The Demise of Rinderpest: The Global Rinderpest Eradication Programme}

In 2011, rinderpest was declared globally eradicated by the United Nation's (UN) Food and Agriculture Organization [FAO] and the World Organisation for Animal Health [OIE]) although the last cases had been seen a decade earlier; thus, it became the second disease for which global eradication had been achieved, after smallpox. This extraordinary achievement was the successful outcome of the Global Rinderpest Eradication Programme (GREP) which commenced in the 1980s under the auspices of FAO in close collaboration with other international organisations and the invaluable assistance of a number of donors including the European Community. Whilst GREP provided strong international co-ordination and leadership, linking constantly with regional bodies such as the African Union's Inter-African Bureau of Animal Resources, national authorities in rinderpest affected countries had executed practically all control and eradication activities.

\section{Some Factors Favouring Success IN RiNDERPEST ERADICATION}

Although not the only tool, vaccination was an important element in the eradication process. Available was a safe, effective, and affordable live attenuated vaccine, developed in the 1950s; this provided life-long sterile immunity and 
could be produced in bulk at low cost. Sensitive and reliable diagnostic tools for detection of virus antigen and antibodies were also available. For the most part, these were internationally standardised, available in a kit format, and their use was linked to laboratory quality assurance activities including proficiency testing. This approach proved invaluable for disease surveillance and eventually demonstrating freedom from infection. Perhaps most importantly, increasingly the national programmes were driven by a deep and solid understanding of the epidemiology of the disease at local, national, and regional level. Whilst at the commencement of the eradication process, there were gaps in knowledge of the disease and the best way to control and eradicate rinderpest, GREP and its partners ensured that these gaps were resolved through good science, research, and the application of sound epidemiology. Mathematical modelling was employed to help clarify specific epidemiological problems without rigid adherence to these or outdated predecessors.

Given the profound success of this programme and the similarities with some aspects of the current SARS-CoV-2 (COVID-19) pandemic, it seems useful to compare aspects of the current management of this pandemic with that of GREP. It is, of course, appreciated that there are significant differences, yet comparison can possibly provide valuable insights.

\section{COMPARING GREP WITH COVID-19 MANAGEMENT}

Central to the successful control and eradication of rinderpest was strong international leadership through the UN agencies. This provided not only an agreed technical pathway to eradication, but a linking of donor resources and identified needs. Contrast this with the current COVID-19 situation characterised by a lack of international leadership and the diversity of ever-changing national approaches and processes. Funding so far raised to support efforts in less well-off countries has been woefully inadequate and is likely to lead to a legacy of prolonged endemicity in these regions. Few would deny that the COVID-19 pandemic is a global issue, and its termination must be considered a global public good. Unless viral circulation is curbed across the whole global community nowhere can ever be safe from re-introduction or draconian control measures.
Experience of rinderpest eradication demonstrated clearly, inter alia, that essential prerequisites for control and elimination of a contagious pathogen are a safe and highly efficacious vaccine used in combination with a set of fit for purpose, highly specific, and sensitive diagnostic tests guided by an understanding of the determinants of disease occurrence, i.e. disease epidemiology. In our experience, the essence of effective control is to be able to identify, delimit and explain transmission hubs-areas/populations where transmission is occurring ("hotspots")—and to focus the use of an effective vaccine on specific host populations in these hubs rather than mass, area-wide (blanket) vaccination. Experience has demonstrated that the latter is more costly and less effective in raising herd immunity to the exclusion threshold.

Surveillance is a key activity for focussing vaccination. To strengthen this activity, international co-ordination for technology transfer and strategy implementation became a critical issue for GREP. International reference laboratories (The Pirbright Institute, the UK, and CIRAD EMVT in France) provided technical development and technology transfer with the UN supporting international laboratory networks. The OIE provided a mechanism for publishing standards and progressing along an 'OIE Pathway' to verification of rinderpest freedom. In comparison, none of this currently exists for COVID-19.

As examples, one technical outcome was the development and commercialisation of a rapid lateral flow immunochromatographic diagnostic test. This became invaluable in the later stages of rinderpest eradication, and indeed, the last cases of rinderpest in Pakistan and Kenya were diagnosed using such a test. Innovative approaches to surveillance using participatory rural appraisal techniques were developed to involve communities in surveillance invaluable in demonstrating freedom from rinderpest.

Compare this to COVID-19 testing, with its apparent devotion to PCR testing of dubious efficacy, despite the availability of rapid patient-side tests.

The prevention of severe disease and death is an important objective but more important in the control process, and essential for eventual elimination of the virus from a population, is the prevention of infection and onward transmission. It seems that none of the vaccines currently made available for COVID control comply with these conditions. Indeed, currently available vaccines are far from ideal; they do not provide adequate protection from, nor prevent onward transmission of, infection. It is difficult to see how their use can be expected to effectively control 
and even eliminate infection in a population and the longer their suboptimal use goes on the more chance there is of mutant viruses (variants) emerging and being able to escape immune control.

During GREP, alternative vaccines did emerge, but the temptation to use these was resisted when there was a tried and tested vaccine available. Surprisingly, with COVID-19, the development of vaccines has concentrated on the mRNA vaccine platform, the products of which are to a large degree clinically unproven despite the unprecedented speed of their development (Abbasi 2020) and licensing. There are serious questions over the degree and duration of immunity produced, the spectrum and duration of protection, and even their safety. It is open to question why a more conventional approach to vaccine development was not addressed initially while exploring the mRNA vaccine platform. Despite statements to the contrary about the unavailability of animal coronavirus vaccines, there is an extensive body of knowledge testifying to the use of vaccines to manage coronavirus diseases in domesticated animals (Tizard 2020).

Through GREP, and adopted by most participating countries, was a strong communication programme that provided regular reports on the progress towards eradication submitted nationally and internationally for assessment. Messaging was shared across countries with a common approach to recognising rinderpest, to ensuring a cold chain for vaccine supply, and even to identifying vaccinated cattle (a clover-leaf ear punch). The OIE pathway provided clear and measurable targets towards eradication with the end goal of a national declaration of freedom from the virus with an independent verification process. Compare this to COVID-19 with a bewildering array of messages and communication on vaccines, on lockdowns, on facemask use, and so on. Even within countries, communication is often confusing and contradictory providing little confidence to the public that a rational and effective process is in place that can be expected to work for COVID-19 control. Government briefings and newspaper articles show little sign that even basic concepts of disease control and elimination have been learnt.

Similar to other beta-coronaviruses (e.g. severe acute respiratory syndrome [SARS-CoV 1] and middle east res- piratory syndrome virus) SARS-CoV-2 appears to have crossed the species barrier, most likely from bats, clearly reinforcing the One Health concept. This concept was developed in recognition of the importance of collaboration between veterinarians and physicians to help solve global health problems. Yet, veterinarians have been almost totally excluded from the decision-making process in COVID control even though veterinary medicine deals with many communicable diseases.

Several authors have argued that it is irrational to exclude veterinarians from COVID-19 control (Roeder 2020; Sibley and Brownlie 2020) and include stating that veterinarians "would not manage COVID-19 this way" instead build on their knowledge of the principles of biosecurity, biocontainment, surveillance, and resilience. To not benefit from this expertise for the control of Covid-19 is irrational at a time when it has become abundantly clear that we need to make use of all available resources to overcome this global crisis.

In conclusion, it does appear that COVID-19 management decision making has been driven by political expediency and a fiscal frenzy relating to the provision of inputs rather than good science. Scant heed seems to have been given to lessons learnt from previous disease control programmes. Unless things change, the outcomes of COVID-19 control are not likely to be the success that characterized rinderpest eradication.

\section{REFERENCES}

Abbasi J (2020) COVID-19 and mRNA Vaccines-First Large Test for a New Approach. JAMA. 324(12):1125-1127. https:// doi.org/10.1001/jama.2020.16866

Roeder P (2020) It is irrational to exclude vets from Covid-19 control. Veterinary Record Letters and Notices 30 May/6 June 2020 doi: https://doi.org/10.1136/vr.m2020

Roeder PL, Mariner J, Kock R (2013) Rinderpest: the veterinary perspective on eradication. Philos Trans R Soc Lond B Biol Sci 368 (1623) doi: https://doi.org/10.1098/rstb.2012.0139

Sibley D, Brownlie J (2020) Vets would not manage COVID-19 this way. Veterinary Record 18/25 April 2020 doi: https://doi. org/10.1136/vrm3004

Tizard IR (2020) Vaccination against coronaviruses in domestic animals. Vaccine 38(33):5123-5130. https://doi.org/10.1016/ j.vaccine.2020.06.026 